CAHIERS DE

NARRATOLOGIE

\section{Cahiers de Narratologie}

Analyse et théorie narratives

$36 \mid 2019$

Rhétorique et représentations de la culture mafieuse.

Images, rituels, mythes et symboles

\title{
« De la force d'attraction des récits de fiction ». Entretien avec Vincent Jouve
}

À propos de Pouvoirs de la fiction. Pourquoi aime-t-on les histoires?, Paris, Armand Colin, 2019, " La Lettre et l'Idée »

Propos recueillis par Frank Wagner

\section{Frank Wagner}

\section{(2) OpenEdition}

\section{Journals}

Electronic version

URL: http://journals.openedition.org/narratologie/10136

ISSN: $1765-307 X$

Publisher

LIRCES

Electronic reference

Frank Wagner, « «De la force d'attraction des récits de fiction ». Entretien avec Vincent Jouve »,

Cahiers de Narratologie [Online], 36 | 2019, Online since 20 December 2019, connection on 21

December 2019. URL : http://journals.openedition.org/narratologie/10136

This text was automatically generated on 21 December 2019.

Article L.111-1 du Code de la propriété intellectuelle. 


\section{« De la force d'attraction des récits de fiction ». Entretien avec Vincent}

\section{Jouve}

À propos de Pouvoirs de la fiction. Pourquoi aime-t-on les histoires?, Paris, Armand Colin, 2019, « La Lettre et l'Idée »

Propos recueillis par Frank Wagner

\section{Frank Wagner}

$\left.11^{\circ}\right)$ Frank Wagner : Vincent Jouve, dans l'introduction de votre dernier ouvrage en date, Pouvoirs de la fiction, après avoir pris acte du considérable succès public que rencontrent aujourd'hui les récits de fiction, tous médias confondus, vous vous fixez comme objectif de " comprendre d'où vient cette force d'attraction du narratif " (p. 7). À l'échelle de votre bibliographie, on peut donc éprouver l'impression que vous êtes graduellement passé du "comment ?", à l'époque de La Poétique du roman (Armand Colin, 1997) ou de Poétique des valeurs (PUF, 2001) par exemple, au " pourquoi ? ", dès Pourquoi étudier la littérature? (Armand Colin, 2010). Quel regard portez-vous personnellement sur cette évolution, qui paraît en phase avec celle de nombre de recherches en poétique du récit?

2 Vincent Jouve: La question du «comment?»(fondamentale) suppose résolue la question du «pourquoi? ?. Or, ce n'est plus le cas aujourd'hui. Nous sommes, comme vous le savez, dans une crise de légitimité des études littéraires. Après m'être interrogé sur ce que peut apporter l'enseignement des lettres dans Pourquoi étudier la littérature?, j'en suis logiquement venu à me pencher sur les raisons qui nous poussent à consommer des textes littéraires et, plus généralement, des fictions. Comme le font remarquer les tenants du «darwinisme littéraire » (un courant théorique qui tente de replacer le fait littéraire et artistique dans le schéma de l'évolution), il est tout de même étrange que l'être humain ait consacré autant de temps à inventer et raconter des histoires au lieu de chasser et de se reproduire. Pourquoi avons-nous besoin de récits imaginaires? Que nous apportent les textes de fiction? L'espoir, c'est, qu'une fois 
ces questions résolues, on puisse en revenir à l'analyse des textes. Mais à une analyse " enrichie ", qui prendrait en compte leur mode d'action sur le lecteur.

$\left.2^{\circ}\right) \mathrm{FW}$ : Cet infléchissement engage bien évidemment une forme d'aggiornamento épistémologique. Sans pour autant renier en rien vos travaux antérieurs de poétique et/ ou de théorie de la lecture, vous soulignez vous-même la nécessité, pour tenter de répondre aux questions que vous vous posez désormais, d'associer diverses "méthodes " (p. 8). Vous serait-il possible de revenir brièvement sur ces options méthodologiques comme sur leurs enjeux?

$4 \quad$ VJ : A partir du moment où on ne parle plus seulement des textes mais du fait littéraire ou du fait fictionnel, on est face à une donnée anthropologique qui ne peut être cernée qu'en mobilisant l'ensemble des sciences humaines. Notre besoin de fiction tient à des raisons psychologiques, culturelles, sociales, mais peut-être aussi biologiques. Pour répondre à la question "pourquoi ne quitte-t-on pas la salle en courant quand on a peur au cinéma?"», on ne peut pas se contenter de dégager la structure du film, voire les procédés qui suscitent la peur. Il faut aussi réfléchir sur la différence entre la peur ressentie dans la réalité et la peur suscitée par une œuvre de fiction, et - surtout expliquer pourquoi un certain nombre de spectateurs aiment avoir peur. L'esthétique ne nous dit pas grand-chose sur ces questions. En revanche, l'anthropologie, les sciences cognitives, voire les théories de l'évolution, nous donnent un certain nombre d'instruments.

$\left.53^{\circ}\right) \mathrm{FW}$ : En termes de méthode(s) toujours, à l'instar du Raphaël Baroni de Les Rouages de l'intrigue. Les outils de la narratologie postclassique pour l'analyse des textes littéraires (Slatkine, 2017), vous vous déclarez " convaincu qu'une bonne théorie se juge à son application" (p. 9). Pourriez-vous élucider cette "conviction", que tous les théoriciens ne partagent pas nécessairement, et qui, dans votre cas, se révèle d'autant plus importante qu'elle conditionne l'existence même du dernier chapitre de votre livre («5. Applications »)?

6 VJ : Même si l'évolution de mes questionnements m'a conduit à sortir du strict champ de ma discipline, je reste fondamentalement un professeur de littérature. Dès lors, une théorie ne m'intéresse que si elle peut, in fine, me donner de nouveaux outils pour l'analyse des textes. Sinon, on en reste à des modèles très généraux qui peuvent intéresser la philosophie et les sciences humaines mais dont, en tant que littéraires, on ne sait pas trop quoi faire. Pendant mes études, on m'enseignait les grands modèles théoriques des années 1970 : ceux du groupe Tel Quel, de Kristeva, de Sollers. C'étaient des machines intellectuelles fascinantes, mais dès qu'on se demandait comment les appliquer aux textes, on était un peu perdu. C'était pour moi une grande frustration. J'en ai gardé la conviction qu'on pouvait (devait) théoriser, mais sans jamais perdre de vue l'objectif final pour nous, littéraires : renouveler notre regard sur les textes. C'est ce que j'ai tenté de faire.

$\left.7 \quad 4^{\circ}\right) \mathrm{FW}$ : Puisqu'il vient d'être question de l'architecture de votre ouvrage, on peut, sans dommage pour sa lecture, je crois, préciser que vous y abordez successivement les problématiques de l'intérêt, de l'émotion et du sentiment esthétique, avant de vous intéresser aux gratifications à plus long terme que lecteurs et lectrices peuvent retirer de leur activité. Ce plan repose pour partie sur une dissociation de l'intérêt et de l'émotion, ou si l'on préfére du cognitif et de l'affectif, ce qui vous conduit notamment à souligner la dimension épistémique de la surprise, du suspense et de la curiosité, qu'à la suite de Meir Sternberg, Raphaël Baroni présente pour sa part (dans La Tension 
narrative, Seuil, 2007) comme autant de "fonctions thymiques». Vous serait-il possible de justifier ce réajustement?

VJ : C'est un des points de mon essai que je considère comme les plus importants. Pour rendre à César ce qui revient à César, c'est un psychologue cognitiviste, J.-L. Dessalles, qui travaille sur les conversations quotidiennes, qui a attiré mon attention sur ce point. Quand on aime discuter avec quelqu'un, ce n'est pas forcément parce que ses propos nous touchent; ce peut être, plus banalement, parce qu'il réussit à susciter notre intérêt. Il me semble qu'on peut élargir cette distinction à la pratique de la lecture. Un récit peut m'émouvoir mais aussi, tout simplement, m'intéresser. C'est quelque chose que R. Baroni a très bien vu en soulignant l'importance de la curiosité, du suspense et de la surprise, qui ont une dimension fortement épistémique. Mais lorsqu'on a repris sa distinction, on a souvent parlé d'« émotions " narratives (j'ai moi-même dû le faire dans le passé). Ce n'est qu'une question de terminologie ; mais, comme l'intérêt d'un récit ne repose pas sur les mêmes ressorts que son impact en termes d'émotion (ce que je me suis efforcé de montrer), il m'a semblé préférable d'utiliser des mots différents.

$\left.5^{\circ}\right) \mathrm{FW}$ : En outre, vous montrez clairement que l'intérêt narratif est dans la plupart des cas fonction de l'inattendu à quoi lecteurs et lectrices se trouvent confrontés; ce qui, de prime abord, paraît relever du bon sens. Toutefois, vous insistez également sur le fait que cette question de l'inattendu et de l'intérêt subséquent ne se pose pas tout à fait dans les mêmes termes dans les récits fictionnels et factuels; soulignez la nécessité de distinguer inattendu diégétique et esthétique; enfin pointez les "limites de l'analyse en termes d'inattendu " (p. 41 sq.). Cela fait trois questions en une, j'en ai peur, mais pourriez-vous évoquer succinctement ces divers points?

10 VJ : L'inattendu est en effet au fondement de la séduction narrative. Rien de pire qu'un récit entièrement prévisible! Mais, dans le récit de fiction, l'inattendu ne concerne pas uniquement l'enchaînement des faits ; il peut aussi porter sur la façon d'écrire. Quand un romancier (ou un cinéaste) invente une nouvelle technique, une nouvelle façon de raconter, il provoque la plupart du temps un sentiment de surprise, qui peut aller jusqu'à l'émerveillement : « on ne m'avait jamais raconté une histoire comme ça!», se réjouit le lecteur. Cela peut suffire à donner envie de lire la suite. Maintenant, qu'il s'agisse du contenu ou de l'écriture, tout ne se ramène pas à l'inattendu dans un récit. L'intérêt peut aussi tenir à la complexité. Une situation complexe n'est pas forcément une situation inattendue: c'est une situation qu'on ne sait pas très bien comment interpréter, qui est susceptible de différentes lectures. Alors que l'inattendu est ponctuel et se trouve en général expliqué dans le cours du récit, le sentiment de complexité peut fort bien demeurer après la lecture. Quand le bossu de Féval révèle qu'il est Lagardère, c'est inattendu sur le moment ; mais le lecteur est assez vite capable de restituer la suite des événements qui ont conduit à ce coup de théâtre. En revanche, je ne sais toujours pas (ou, du moins, pas avec certitude) pourquoi Swann a failli mourir pour une femme qui, de son propre aveu, n'était pas son genre.

$\left.116^{\circ}\right) \mathrm{FW}$ : Même si les travaux de Michel Picard (La Lecture comme jeu, Minuit, 1986) et les vôtres (L'Effet-personnage dans le roman, PUF, 1992 ; La Lecture, Hachette, 1993) nous ont enseigné que la lecture littéraire est loin de se limiter à la mise en cuvre des seules facultés cérébrales, il n'empêche que lire, sur le plan cognitif, c'est tout de même interpréter, ou tenter de le faire. Précisément, certaines de vos analyses, fondées sur les recherches en sciences cognitives, portent sur "l'intérêt herméneutique " (p. 44 sq.), appréhendé en termes de "complexité " (id.). Vous serait-il dès lors possible de nous 
donner un aperçu de la façon dont, à la lecture d'un récit de fiction, s'articulent selon vous " complexité " et " accessibilité " ?

$\mathrm{VJ}$ : Le problème, avec la complexité, c'est qu'elle peut fasciner mais aussi rebuter le lecteur si elle dépasse un certain seuil. Si un récit est trop complexe, le risque, c'est que je finisse par le trouver opaque, voire franchement hermétique et qu'en conséquence la communication ne fonctionne plus. Pour «intéresser», la complexité doit donc toujours être accessible. Il ne faut jamais oublier que le consommateur de fiction recherche avant tout le plaisir: il ne faut donc pas exiger de lui trop d'efforts. Si j'annonce que j'ai trouvé un lithodes santolla (crabe royal de Patagonie) de couleur brune, la plupart des gens ne comprendront pas l'intérêt de cette découverte tout simplement parce qu'ils ignorent tout du lithodes santolla (et, en particulier, qu'il est réputé pour sa couleur rouge vif). En termes de plaisir de lecture, une situation complexe ne peut dégager toute sa puissance que si le lecteur n'a aucun mal à se la représenter. Si «Un amour de Swann » est si célèbre, c'est parce que personne n'a de mal à se représenter une histoire de passion amoureuse. En d'autres termes, pour que la fiction fonctionne comme fiction, il faut éviter que la complexité nuise à l'immersion ; l'histoire doit toujours demeurer accessible.

$\left.13 \quad 7^{\circ}\right)$ FW : Après avoir traité ces épineuses questions, vous soulignez que "avec l'intérêt, l'émotion est l'autre grand moteur de la séduction narrative " (p. 61), ce qui ne paraît guère souffrir de contestation - surtout compte tenu du "tournant affectif" que d'aucuns identifient à l'heure actuelle dans les études littéraires. Mais l'" évidence " de ce paramètre n'est là encore qu'illusoire. Ainsi, après avoir rappelé à quel point il peut être délicat de définir les différents types d'émotions, vous soulignez la spécificité de celles que dispense la fiction, et vous attachez enfin plus particulièrement au repérage des facteurs susceptibles d'entraîner des variations de l'intensité émotionnelle (p. 80 sq.) dans le cadre de l'activité de lecture. Pourriez-vous dès lors brièvement expliquer en quoi l'on peut considérer que la lecture de récits de fiction dispense des émotions spécifiques? Et préciser comment les paramètres de la proximité, de l'improbabilité et de la gradualité influent sur l'intensité émotionnelle dont lecteurs/lectrices font l'expérience?

14 VJ : En vérité, la fiction ne dispense pas d'émotions spécifiques. Quand on est triste pour un personnage, on est vraiment triste ; et, quand on est content, on est vraiment content. Comme l'a montré Schaeffer, c'est dans le traitement que nous faisons des émotions que réside la différence. Dans la vie réelle, les émotions nous conduisent à adopter tel ou tel comportement. Mais, dans le cadre de la fiction, on se contente de les ressentir (Thénardier est un sale type, mais, comme je ne peux rien faire pour sauver Cosette, je me contente de tourner les pages des Misérables). C'est justement là le point important : à la lecture, je peux "vibrer ", autrement dit vivre plus intensément, en toute sécurité, sans craindre que ce flux d'émotions me conduise à des comportements inadaptés (si l'on excepte le cas, pathologique, des lecteurs confondant fiction et réalité). Bref, j'ai le "bon » côté des émotions sans les "mauvais ». L'émotion est essentiellement liée à la situation représentée (ces malheureux passagers du Titanic qui sont en train de se noyer !), mais les récits - et, ici, on retrouve l'analyse textuelle peuvent, par une série de procédés, «intensifier » ces émotions : on sera d'autant plus facilement ému par une situation qu'elle concerne des personnages que le récit nous a rendus "proches", qu'elle nous apparaît comme injuste (elle n'aurait pas dû se 
produire) et qu'elle a franchi un certain seuil (perdre dix euros au jeu, ce n'est pas très grave ; mais perdre ses économies, sa voiture, sa maison...).

$\left.8^{\circ}\right) \mathrm{FW}$ : À vous suivre, en matière de récit de fiction, le $3^{\text {ème }}$ facteur décisif de séduction réside dans sa capacité de produire de l'intérêt esthétique - ce qui paraît difficilement contestable. L'intérêt majeur de ce chapitre réside selon moi dans la distinction qu'à partir des réflexions de Jean-Marie Schaeffer (Les Célibataires de l'art. Pour une esthétique sans mythes, Gallimard, 1996), vous opérez entre plaisir esthétique et plaisir fictionnel, ainsi - et à vrai dire surtout - que dans votre hypothèse d'une tension entre immersion fictionnelle et satisfaction esthétique. Pourriez-vous en éclairer les modalités, et dire deux mots des enseignements qu'il paraît possible d'en tirer?

VJ : Quand je propose cette distinction, j'emploie le mot «esthétique» dans son sens classique de "plaisir suscité par la dimension artistique d'un objet d'art» (texte bien écrit, film bien construit, etc.). Or, le récit de fiction est aussi un objet d'art. Il suscite donc, lorsqu'il est réussi, deux plaisirs assez différents : le plaisir de s'immerger dans une histoire (je m'intéresse à ce qui arrive aux personnages, aux événements qui se produisent) et le plaisir de "consommer " un objet agréable dont les phrases sonnent bien, dont le style est fluide, qui éventuellement peut me surprendre par des innovations formelles. Le problème est que ces deux dimensions peuvent entrer en conflit: l'immersion dans l'histoire suppose que j'oublie momentanément que j'ai affaire à un texte alors que le travail sur l'écriture, s'il est trop visible, me rappelle que j'ai affaire à un texte. Le risque, si c'est trop bien écrit, c'est qu'on n'y croie plus ! Barthes disait : j'ai une maladie, je vois le langage. Je ne sais pas si on peut en tirer des enseignements. Disons qu'en tant qu'écrivain, il faut savoir quelle est sa priorité : écrire un «beau » texte ou raconter une histoire prenante. Mais les «très grands » auteurs arrivent à concilier les deux, comme, par exemple, Flaubert dans Madame Bovary.

$\left.9^{\circ}\right) \mathrm{FW}$ : Votre 4ème chapitre, "Les vertus de la fiction" (p. 111 sq.) peut paraître déterminant, dans la mesure où vous y abordez frontalement ces pouvoirs évoqués dans le titre de votre ouvrage. C'est en effet une fois parvenu à ce stade de votre raisonnement que, dans le sillage d'un Ricœur (Le Conflit des interprétations, Seuil, 1969) réfléchissant à la "signification" conçue comme moment de la reprise du sens par le lecteur, de son effectuation dans l'existence réelle, vous scrutez les bénéfices que lecteurs et lectrices peuvent retirer de leur activité. Vous serait-il dès lors possible de donner un aperçu de ces somme toute nombreux pouvoirs du récit de fiction?

VJ : Il me semble peu contestable que le récit de fiction ait de nombreux "pouvoirs ", ou " effets", si l'on préfère. Outre sa fonction compensatrice évidente (il nous permet de réaliser imaginairement toute une palette de désirs), il nous permet d'expérimenter les situations les plus diverses (au moins sur le plan émotionnel) en nous évitant les écueils d'un apprentissage dans le monde réel. Il nous permet ainsi de découvrir des dimensions de nous-mêmes que, sans le relais de la fiction, nous n'aurions jamais eu l'occasion d'actualiser. La fiction permet aussi de se mettre dans la peau de personnages qui ne nous ressemblent pas, ce qui est très formateur en termes d'ouverture à l'altérité. De ce point de vue, on peut dire que la lecture de fiction participe au renforcement du lien social.

10) FW : En conclusion de ce chapitre essentiel, analysant, en matière de développement cognitif, le poids relatif du texte et du travail interprétatif que lecteurs/lectrices effectuent sur lui, vous prenez la précaution de souligner l'importance de la qualité de l'objet sur lequel se porte le regard. Dans la mesure où votre position prend le contrepied 
de celle qui tend bien souvent à prévaloir de nos jours dans le champ des études culturelles, comment justifieriez-vous votre insistance sur la qualité ou la valeur esthétique du corpus?

$\mathrm{VJ}$ : Avec le développement des études culturelles (qui me paraît positif en lui-même : on a tout intérêt à savoir comment fonctionnent les objets culturels que nous consommons massivement), la tendance est de considérer que tous les objets culturels se valent (leur hiérarchie en termes de qualité relevant, nous dit-on, d'un point de vue subjectif et culturel). L'important, affirment les culturalistes (dont certains sont excellents), c'est le regard que l'on porte sur l'objet : un angle d'approche approfondi peut rendre n'importe quel objet intéressant. Je pense que non. On peut certes dire des choses passionnantes sur le steak frites (voyez Barthes dans Mythologies); mais on tourne très vite en rond. Alors que, lisant Hamlet ou Don Quichotte, on peut, quasiment à chaque lecture, découvrir de nouveaux sens ou centres d'intérêt. Ce « constat » (ou ce que je considère comme tel) est pour moi fondamental: c'est lui qui justifie la spécificité des études littéraires. Ce qui n'implique en aucun cas qu'on ne doive pas pratiquer par ailleurs des études culturelles. Ce qui me semble dangereux, c'est la dissolution des premières dans les secondes.

$\left.211^{\circ}\right) \mathrm{FW}$ : En matière de valeur esthétique, les textes auxquels vous consacrez les études empiriques de détail constituant votre $5^{\text {ème }}$ et dernier chapitre (La Fortune des Rougon de Zola, Albertine disparue de Proust, Le Vice-consul de Duras) devraient sans trop de peine pouvoir faire l'objet d'un consensus... Dans la mesure où vous les avez visiblement sélectionnés afin de vous confronter à des "échantillons " esthétiquement variés, vous serait-il possible de synthétiser les "pouvoirs" (partagés, ou, à l'inverse, singuliers) qu'ils vous ont paru manifester?

VJ : J'ai volontairement choisi des «classiques» de la littérature pour montrer que la force des classiques est précisément dans ce pouvoir qu'ils ont de continuer à nous parler. Comme je vous l'ai dit, je ne crois pas que tous les objets aient la même valeur. De façon un peu mécanique (dans un souci didactique, on ne se refait pas!), j'ai appliqué aux trois extraits la même méthode pour voir ce qu'elle pouvait donner sur des textes de factures très différentes. L'exercice comporte à la fois une dimension poéticienne (par quels procédés tel passage suscite-t-il de l'intérêt et de l'émotion ?) et une dimension inévitablement plus personnelle puisque les "effets" produits par un texte sont en grande partie fonction de l'équation propre à chaque lecteur. J'ai donc pris mes réactions à titre d'exemple. Elles n'ont pas de valeur générale : mon objectif était de montrer comment, dans la lecture, le récit se mêle à la texture intime de chacun. Mais ce peut être une façon d'attirer vers les textes un public qui a tendance à s'en éloigner: si on veut (re)donner l'envie de lire aux adolescents, leur montrer comment, à partir de procédés objectifs, le texte suscite une expérience singulière qui les concerne au plus profond n'est sans doute pas la plus mauvaise des stratégies.

12') FW : À la faveur d'un ultime clin d'œil à l'œuvre de Proust, vous concluez votre ouvrage par une forme d'hommage à ce que, "faute d'un terme plus adéquat ", écrivezvous (p. 188 et dernière), vous qualifiez de richesse "intérieure" (idem) "alimentée " par les récits - par-delà le plaisir, aussi intense soit-il, suscité par leur apparence. Pourriez-vous quelque peu préciser ce que peut être cette "richesse intérieure " que la fréquentation de certains récits de fiction nous permettrait d'accroître?

24 VJ : Le récit doit d'abord séduire sur le plan formel. C'est la condition préalable pour que nous acceptions de continuer l'aventure et de vivre des expériences plus 
profondes, c'est-à-dire qui nous touchent « intérieurement », dans ce que nous pensons ou ressentons. C'est un peu ce que dit Proust pour la rencontre amoureuse : Marcel remarque les yeux d'Albertine (qui n'est, pour l'heure, qu'une cycliste croisée sur la plage). Mais il explique que si ce regard le retient, ce n'est pas seulement parce que c'est un beau regard - un regard lumineux -, mais parce qu'il ouvre sur la richesse intérieure de la jeune femme, sentiment qui lui donne envie de la connaître plus avant.

13) FW : Vincent Jouve, pour clore cet entretien, permettez-moi une ultime question. Les "pouvoirs" de la littérature, et en son sein du récit de fiction, sont fréquemment abordés dans une perspective non pas anthropologique, comme la vôtre, mais disons "socioculturelle". La question est alors de savoir ce que peut le récit fictionnel à une échelle supra-individuelle. Telle n'était bien sûr pas votre perspective dans le cadre de cet ouvrage, mais à titre de prolongement, et peut-être de complément, que pensez-vous de ces autres (?) pouvoirs de la fiction?

$\mathrm{VJ}$ : Je n'ai pas abordé la dimension socioculturelle dans cet essai car je voulais réfléchir sur le fait narratif en tant que tel. On consomme des récits dans toutes les cultures et depuis un temps immémorial, sans doute depuis les débuts de l'humanité. Je me suis donc situé sur un plan forcément très général. Mais il est évident que les principes généraux de la séduction narrative se déclinent en termes sociologiques et culturels. C'est pour cela qu'il y a une histoire de la littérature et du récit. Il me semble toutefois que l'intérêt du corpus littéraire est dans cette capacité qu'il a de nous arracher à nos repères (historiques, culturels, sociologiques) pour nous ouvrir à de nouvelles façons de voir et de penser. On peut certes faire étudier aux adolescents d'aujourd'hui des produits qu'ils consomment déjà et qui correspondent parfaitement à leurs attentes (films à grand spectacle, séries télévisées, bandes dessinées). Comme je l'ai dit, cela présente l'intérêt de les conduire à adopter un regard réflexif sur leurs propres pratiques. Mais il me semble que leur faire aimer des textes qui traitent de questions qu'ils ne se posaient pas forcément, et dans des termes qu'ils n'imaginaient pas forcément, est infiniment plus enrichissant. 